\title{
Fabrication of gelatin methacrylate/ nanohydroxyapatite microgel arrays for periodontal tissue regeneration
}

This article was published in the following Dove Press journal:

International Journal of Nanomedicine

14 September 2016

Number of times this article has been viewed

Xi Chen'

Shizhu Bai'

Bei $\mathrm{Li}^{2}$

Huan Liu'

Guofeng $\mathrm{Wu}^{\prime}$

Sha Liu $^{3}$

Yimin Zhao'

'State Key Laboratory of Military Stomatology and National

Clinical Research Center for Oral Diseases, Shaanxi Key Laboratory of Oral Diseases, Department of Prosthodontics, ${ }^{2}$ State Key Laboratory of Military Stomatology and National Clinical Research Center for Oral Diseases, Shaanxi Key Laboratory of Oral Diseases, Center for Tissue Engineering, School of Stomatology, ${ }^{3}$ Department of Plastic and Reconstructive Surgery, Xijing Hospital, The Fourth Military Medical University, Shaanxi, People's Republic of China
Correspondence: Yimin Zhao State Key Laboratory of Military Stomatology and National Clinical Research Center for Oral Diseases, Shaanxi Key Laboratory of Oral Diseases, Department of Prosthodontics, School of Stomatology, The Fourth Military Medical University, Changle West Road No 169, Xi'an 7I0032, Shaanxi, People's Republic of China

Tel +8684776465

Fax +868477 6465

Email zhaoymfmmu@I26.com
Introduction: Periodontitis is a chronic infectious disease and is the major cause of tooth loss and other oral health issues around the world. Periodontal tissue regeneration has therefore always been the ultimate goal of dentists and researchers. Existing fabrication methods mainly focused on a top-down tissue engineering strategy in which several drawbacks remain, including low throughput and limited diffusion properties resulting from a large sample size. Gelatin methacrylate (GelMA) is a kind of photocrosslinkable and biocompatible hydrogel, with the capacities of enabling cell encapsulation and regeneration of functional tissues. Here, we developed a novel method to fabricate GelMA/nanohydroxylapatite (nHA) microgel arrays using a photocrosslinkable strategy. The viability, proliferation, and osteogenic differentiation and in vivo osteogenesis of human periodontal ligament stem cells (hPDLSCs) encapsulated in microgels were evaluated. The results suggested that such microgels provide great potential for periodontal tissue repair and regeneration.

Methods: Microgel arrays were fabricated by blending different weight ratios of GelMA and nHA. hPDLSCs were encapsulated in GelMA/nHA microgels of various ratios for a systematic evaluation of cell viability, proliferation, and osteogenic differentiation. In vivo osteogenesis in nude mice was also studied.

Results: The GelMA/nHA microgels exhibited appropriate microarchitecture, mechanical strength, and surface roughness, thus enabling cell adhesion and proliferation. Additionally, the GelMA/nHA microgels $(10 \% / 2 \% \mathrm{w} / \mathrm{v})$ enhanced the osteogenic differentiation of hPDLSCs by elevating the expression levels of osteogenic biomarker genes, such as $A L P, B S P, O C N$, and $R U N X 2$. In vivo ectopic transplantation results showed that GelMA/nHA microgels $(10 \% / 2 \% \mathrm{w} / \mathrm{v})$ increased mineralized tissue formation with abundant vascularization, compared with the $1 \%, 3 \%$, and the pure GelMA group.

Conclusion: The GelMA/nHA microgels $(10 \% / 2 \% \mathrm{w} / \mathrm{v})$ facilitated hPDLSCs viability, proliferation, and osteogenic differentiation in vitro and further promoted new bone formation in vivo, suggesting that the GelMA/nHA microgels $(10 \% / 2 \% \mathrm{w} / \mathrm{v})$ provide great potential for periodontal tissue regeneration.

Keywords: periodontal ligament stem cells, gelatin methacrylate, nanohydroxyapatite, microgel array, differentiation, periodontal tissue regeneration

\section{Introduction}

Periodontitis is an infectious disease involving multiple degenerative and inflammatory states of periodontal supporting structures (ie, alveolar bone, periodontal ligaments [PDLs], and cementum) and is the major cause of tooth loss and oral health issues in the world. ${ }^{1}$ According to the World Health Report in 2013, the total proportion of periodontal patients in USA and People's Republic of China reached $60 \%$ and 90\%, 
respectively. ${ }^{2}$ For seriously damaged or missing periodontal supporting structures, traditional treatment procedures (eg, scaling and root planning, open flap debridement, and injectable drug delivery) can hardly be used for reconstruction owing to the large size of the damaged area and the limited regeneration capacity of such tissues. ${ }^{3}$ The periodontal tissue engineering strategy has emerged as a series of temporal and spatial sequential procedures that enhance periodontal regeneration and is, therefore, important to meet the transplantation and implantation demand in certain clinical therapies. $^{4-7}$

For existing tissue engineering strategies, cells are seeded onto a biocompatible scaffold and expected to populate in the scaffold and remodel the local microenvironment by secreting their own extracellular matrix (ECM). ${ }^{7,8}$ Several kinds of scaffolds have been successfully developed for periodontal tissue regeneration. For example, collagen scaffolds with varying porosities $(40 \% \sim 90 \%)$ have been used for PDL repair and regeneration. ${ }^{9}$ The results suggested that scaffolds with porosity higher than $80 \%$ successfully formed PDL-like tissues after 2 weeks of culture. Chitosan scaffolds combined with PDL stem cells (PDLSCs) have also been applied for improvement of osteogenic differentiation and generation of alveolar bone-like structures in vitro. ${ }^{10}$ Despite several thin or avascular tissues, such as skin, bladder, and cartilage, having been successfully engineered through the top-down approach, the fabrication of complex functional tissues with high cell densities and high metabolic requirements still has challenges. This is because the sample is commonly engineered at centimeter levels, which may affect cell functions as a result of limited diffusion properties of nutrients and oxygen, and therefore it can hardly sustain a long-term culture in vitro. In addition, the existing fabrication method is low throughput and the utilized natural scaffolds can hardly meet the sufficient mechanical strength of in vivo periodontal tissues, especially for alveolar bone and PDL regeneration. Thus, it is important to develop novel fabrication strategies and microscale biomaterials with tunable mechanical properties for periodontal tissue engineering in vitro.

Hydrogels have emerged as a kind of soft material with three-dimensional (3D) polymer network, water-absorbability, and tunable properties (eg, physical, chemical, and biocompatible) and have been found to have widespread use in biomedical applications such as tissue regeneration and drug delivery systems. Recently, both natural and synthetic hydrogels composed of micro-/nanostructures (eg, pores and fibrous structure) have been developed to mimic the chemical and physical properties of natural ECM, and these have further been used for periodontal tissue repair and regeneration. For instance, chitosan hydrogels combined with $\beta$-glycerophosphate have been utilized for the improvement of osteogenic differentiation and generation of alveolar bonelike tissue constructs after 2 weeks of culture. ${ }^{11}$ Polyethylene glycol hydrogels that contain cell adhesive RGD sequences have also been used for treating dentoalveolar defects. ${ }^{12}$ Although such hydrogels were regarded as biocompatible and biodegradable biomaterials for reconstructing periodontiumlike tissues, several issues still remain. For example, the currently used large scale (eg, centimeter level) sample size of these hydrogels will limit the diffusion of nutrients and oxygen. Another issue is that the existing method lacks satisfactory revascularization abilities, which is a key requirement for the engineering of functional 3D tissue constructs such as bone, muscle, heart, and liver. ${ }^{13,14}$

Gelatin methacrylate (GelMA) is gelatin modified by methacryloyl (methacrylamide and methacrylate) side groups that has been recently developed for mimicking 3D cell microenvironment and generating 3D functional tissue constructs due to their biocompatibility, photocrosslinkable ability, and mechanical tunability to a reasonable extent. ${ }^{15,16}$ It is worth mentioning that the GelMA hydrogel is easy to be microfabricated through photopatterning, micromolding, self-assembly, and bioprinting strategies. ${ }^{15}$ GelMA hydrogel has also been successfully utilized in several studies for the development of vascular networks in 3D microenvironments. For instance, GelMA hydrogels provided a favorable microenvironment for human umbilical vein endothelial cells to form a confluent monolayer and for MC3T3 cells to differentiate into osteogenic cells. ${ }^{17} \mathrm{~A}$ hybrid biomaterial based on GelMA and gold nanoparticles has also been used to regenerate bone tissues in vitro. ${ }^{18}$ GelMA hydrogel is therefore appropriate for constructing functional tissues in vitro. Herein, we describe a simple, facile, and high-throughput method to fabricate GelMA/nanohydroxylapatite (nHA) microgel arrays. The microstructure and mechanical properties of GelMA/nHA microgels have been characterized. In vitro and in vivo experiments were conducted to test the viability, proliferation, and osteogenic differentiation of human PDLSCs (hPDLSCs) encapsulated in GelMA/nHA microgels and further evaluate their potential applications to repair damaged alveolar bone. A key enabling feature of this method is the high throughput and simplicity of the fabrication process of such functional microgels, which hold great potential for periodontal tissue regeneration applications. 


\section{Material and methods Materials}

Type A porcine skin gelatin powder and methacrylic anhydride were obtained from Sigma-Aldrich Co. (St Louis, MO, USA). Acicular nHA with purity $>99.0 \%$ was obtained from Emperor Nano Material Co. (Nanjing, People's Republic of China). A dialysis tubing with a cutoff point of $12-14 \mathrm{kDa}$ was obtained from Spectrum Labs (Rancho Dominguez, CA, USA). Irgacure 2959 photocrosslinker was obtained from CIBA Chemicals (Basel, Switzerland). 3-(Trimethoxysilyl) propyl methacrylate (TMSPMA) was purchased from SigmaAldrich Co. Printed photomasks were purchased from Qingyi Co. (Shenzhen, People's Republic of China). Polymethyl methacrylate was purchased from Guangyu Co. (Hangzhou, People's Republic of China). Live/Dead viability/cytotoxicity kit was purchased from Thermo Scientific Co. (Rockford, IL, USA). 3-(4,5-Dimethylthiazol-2-yl)-2,5-diphenyl tetrazolium bromide (MTT) assay and 5-bromo-2-deoxyUridine (Brdu) was obtained from Sigma-Aldrich Co. Anti-BrdU antibody and AlexaFluor488-conjugated AffiniPure Goat Anti-Human IgG was obtained from Abcam (Cambridge, UK). Alizarin Red S, $\beta$-glyceraldehyde-3-phosphate, L-ascorbic acid, dexamethasone, and paraformaldehyde were purchased from Sigma-Aldrich Co.

\section{Synthesis of GelMA}

GelMA, a biocompatible and biodegradable hydrogel that is effective for 3D cell encapsulation, was synthesized as described previously. ${ }^{19}$ Briefly, gelatin powder was added to phosphate-buffered saline (PBS) solution at a temperature of $65^{\circ} \mathrm{C}$ and stirred until fully dissolved at $10 \% \mathrm{w} / \mathrm{v}$. Gelatin powder was added to PBS solution at a temperature of $65^{\circ} \mathrm{C}$ and stirred until fully dissolved at $10 \% \mathrm{w} / \mathrm{v}$. Methacrylic anhydride was added to this solution at a rate of $0.8 \mathrm{~mL} /$ minute while stirring at $45^{\circ} \mathrm{C}$ until the target volume was reached. The mixture was then dialyzed against distilled water at $40^{\circ} \mathrm{C}$ for 2 weeks using the cutoff dialysis tubing. The water was changed every day to remove salts and methacrylic acid (Figure 1B). Then, the GelMA solution was lyophilized and stored at $-80^{\circ} \mathrm{C}$ for future use.

\section{Fabrication of GelMA/nHA microgel arrays}

GelMA solution ( $5 \%$ and $10 \% \mathrm{w} / \mathrm{v})$ was mixed with nHA of varying concentration $(1 \%, 2 \%$, and $3 \% \mathrm{w} / \mathrm{v})$ and Irgacure2959 $(0.5 \% \mathrm{w} / \mathrm{v})$, and the composite solution was added in a polymethyl methacrylate mold. Designed photomasks composed of circle arrays with a diameter of $1 \mathrm{~mm}$ were used here. A TMSPMA-treated glass slide with a pre-patterned photomask labeled on it was also covered on the mold and exposed to $2.9 \mathrm{~mW} / \mathrm{cm}^{2}$ ultraviolet (UV) light $(365 \mathrm{~nm}$ ) for 30 seconds (Figure 1A).

\section{Degradability of GelMA hydrogels}

To evaluate the degradability of cell-laden GelMA hydrogels, we prepared the GelMA sample encapsulated with cells at density of $1 \times 10^{6}$ cells $/ \mathrm{mL}$. The degradability profile of GelMA was obtained by calculating the volume of the hydrogel sample with increasing culturing time.

\section{Scanning electron microscopy}

GelMA/nHA hydrogel arrays were left to swell in deionized water overnight, followed by freezing in liquid nitrogen. Then, the frozen hydrogel was lyophilized overnight using a Thermo Scientific freeze dryer. Lyophilized samples were sputter-coated with iridium and imaged with a scanning electron microscope (SEM) (S-4800; Hitachi, Tokyo, Japan).

\section{Mechanical testing of GelMA/nHA microgels}

GelMA/nHA microgels were detached from the slide and tested at a rate of $15 \%$ strain/minute on a Bose 3200 mechanical tester. The Young's modulus was determined as the slope of the linear region corresponding with $10 \%$ strain.

\section{Cell encapsulation}

hPDLSCs were primarily isolated from one human normal third molar extracted for orthodontic reasons, and single cell-derived colonies were obtained by the limiting dilution technique $\left(\mathrm{P}_{0}\right)$ based on previous reports. ${ }^{20,21}$ Written informed consent was obtained from the patient for use of the tissue. hPDLSCs at passage P2-P6 were used in all the following experiments. Briefly, hPDLSCs were trypsinized and resuspended in GelMA hydrogel precursor composed of $0.5 \% \mathrm{w} / \mathrm{v}$ photoinitiator at a concentration of $1 \times 10^{6}$ cells $/ \mathrm{mL} .{ }^{16}$ Microgel units were fabricated by exposing to $2.9 \mathrm{~mW} / \mathrm{cm}^{2} \mathrm{UV}$ light $(365 \mathrm{~nm}$ ) for 30 seconds on TMSPMAtreated glass slides. The glass slides containing microgels were washed with PBS and incubated in culture medium under standard culture conditions. The culture medium was changed every 2 days.

\section{Viability and proliferation of hPDLSCs in GelMA/nHA microgels}

The viability of $\mathrm{hPDLC}$ encapsulated in GelMA/nHA microgels was evaluated by Live/Dead staining and MTT assay. 

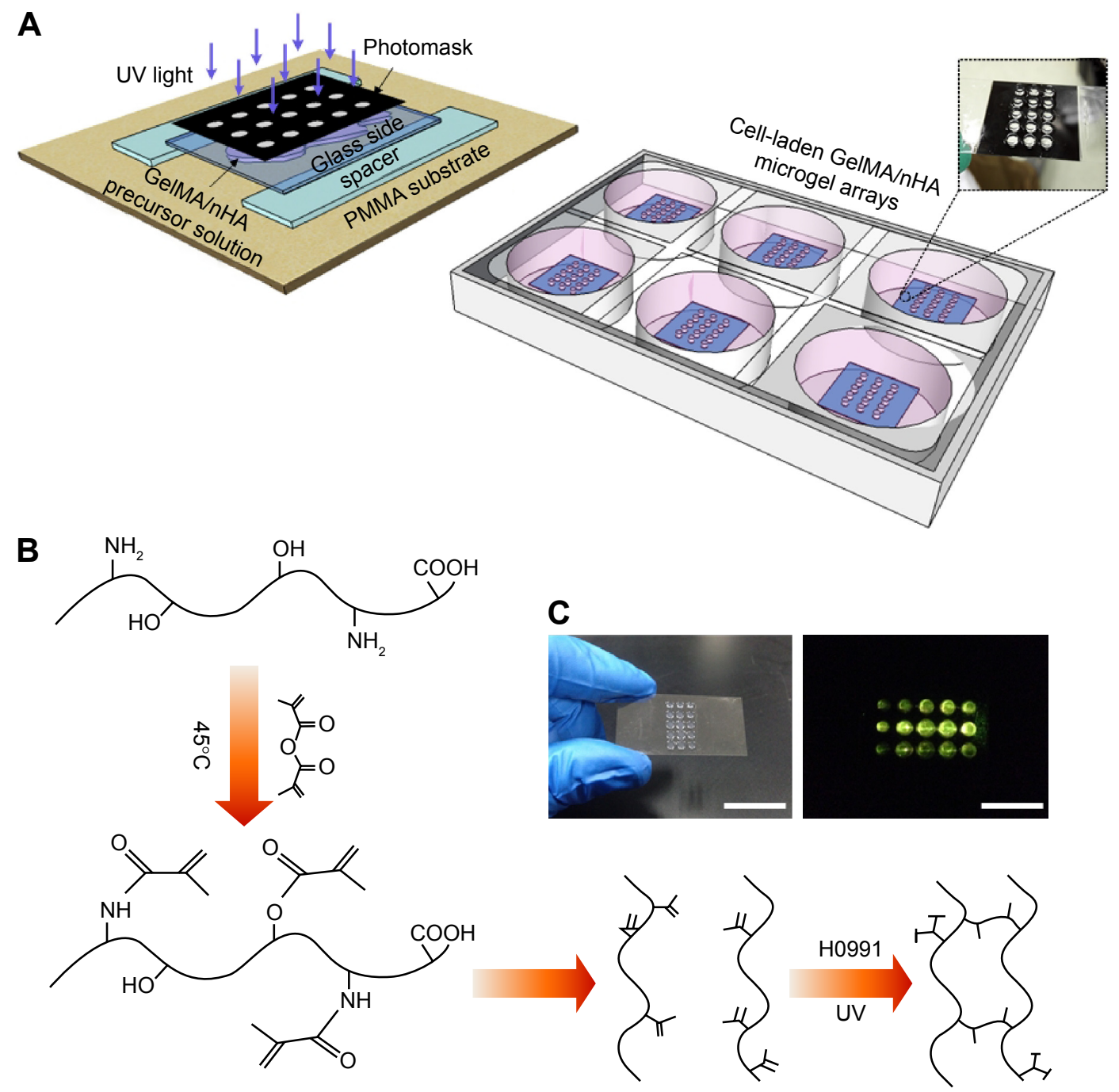

Figure I Schematic representations of GelMA/nHA microgel arrays fabrication method.

Notes: (A) GelMA hydrogel precursor mixed with nHA nanoparticles were polymerized by exposing to UV light through pre-patterned photomask. (B) Synthesis of methacrylated gelatin precursor and gelation of GelMA by UV exposure and photoinitiator. (C) GelMA microgel array. Scale bar: $2 \mathrm{~cm}$ (left), $5 \mathrm{~mm}$ (right).

Abbreviations: GelMA, gelatin methacrylate; nHA, nanohydroxylapatite; PMMA, polymethyl methacrylate; UV, ultraviolet.

For Live/Dead staining, $2 \mathrm{mM}$ ethidium homodimer-1 and $0.5 \mathrm{mM}$ calcein AM were added to the cell-laden microgels in Hank's balanced salt solution for 15-20 minutes. The samples were then imaged using an Olympus IX81 microscope (Tokyo, Japan). MTT assay was also used for quantifying cell viability in GelMA/nHA microgels. The proliferation of hPDLSCs was tested through BrdU incorporation. Briefly, $10 \mu \mathrm{g} / \mathrm{mL}$ BrdU was added to the culture medium and incubated for 12 hours. After washing with Dulbecco's phosphate-buffered saline (DPBS) three times, cells were fixed in 4\% polyformaldehyde for 10 minutes. Then, cells were sequentially incubated in anti-BrdU antibody $(1: 1,000)$ and AlexaFluor488-conjugated Anti-human IgG (1:500). Positive proliferated cells were quantified using fluorescence microscopy.

\section{In vitro osteogenic differentiation of hPDLSCs and Alizarin Red staining}

hPDLSCs-laden GelMA/nHA microgel arrays at a concentration of $1 \times 10^{6}$ cells $/ \mathrm{mL}$ were incubated in osteogenic media (alpha-minimum essential medium supplemented with 10\% fetal bovine serum, $10 \mathrm{mM}$ b-glyceraldehyde-3-phosphate, $60 \mathrm{mg} / \mathrm{mL}$ L-ascorbic acid, and $10 \mathrm{nM}$ dexamethasone) as previously described. ${ }^{22,23}$ The media were changed twice a week, and 4 weeks later, the GelMA/nHA microgel arrays were washed twice with DPBS followed by fixation using $4 \%$ paraformaldehyde for 30 minutes. Then, the cell-laden microgels were incubated in $0.2 \%$ Alizarin Red S stain for 15 minutes. Images were acquired by SEM using an Olympus IX81 microscope. 
Table I Specific primer sequences used for RT-PCR analysis

\begin{tabular}{lllll}
\hline Genes & GenBank number & Forward $\left(\mathbf{5}^{\prime} \mathbf{-} \mathbf{3}^{\prime}\right.$ ) & Reverse (3'-5') & Product size (bp) \\
\hline ALP & NM_001632.4 & AGAATTCCTGCCTCGCCACT & TCTTGGCGGCTGTCTGTGCA & 208 \\
BSP & NM_004967.3 & TGGGGTCTTTAAGTACAGGC & CTTCTGAACTGTCATCTCCA & 128 \\
OCN & NM_199173.5 & TATTGGCCCTGGCCGCACTT & CATTGATACAGGTAGCGCCT & 142 \\
RUNX2 & NM_001278478.1 & ACCAGCAGCACTCCATATCTCTAC & CTTCCATCAGCGTCAACACCATC & 182 \\
$\beta$-actin & NM_001101.3 & CGTCTTCCCCTCCATCGT & GCCTCGTCGCCCACATAG & 87 \\
\hline
\end{tabular}

Abbreviations: RT-PCR, reverse transcription polymerase chain reaction; ALP, alkaline phosphatase; BSP, bone sialoprotein; OCN, osteocalcin; RUNX2, runt-related transcription factor 2 .

\section{Real-time polymerase chain reaction}

Total RNA extraction was performed on hPDLSCs using TRIzol reagent (Invitrogen Life Technology, Carlsbad, CA, USA), and first-strand cDNA syntheses were performed according to the manufacturer's protocol. Reverse transcription was performed using the PrimeScript real-time polymerase chain reaction (RT-PCR) kit (Thermo Scientific) followed by semiquantitative RT-PCR using specific primers. The primer sequences of alkaline phosphatase $(A L P)$, bone sialoprotein $(B S P)$, osteocalcin $(O C N)$, and runt-related transcription factor $2(R U N X 2)$ are shown in Table 1. The expression of the target genes was normalized against the $\beta$-actin. RT-PCR was performed using the 7500 Fast RealTime PCR System (Life Technologies, Inc., Waltham, MA, USA).

\section{In vivo implantation}

Thirty-two 4-6 week male BALB/c-nu/nu mice were purchased from the Fourth Military Medical University Animal Center and housed in a pathogen-free environment $\left(24^{\circ} \mathrm{C}\right.$ and $12 \mathrm{~h} / 12 \mathrm{~h}$ light-dark cycle). The animals were provided with purified water and food ad libitum, and were allowed to acclimatize to local vivarium conditions for at least one week. All animal procedures were performed in accordance to the criteria outlined in the Guide for the Care and Use of Laboratory Animals prepared by the National Academy of Sciences. ${ }^{24}$ The study was approved by the Institutional Review Board of the Fourth Military Medical University School of Stomatology. The nude mice were anesthetized with $2 \%$ inhaled isoflurane and ventilated using a rodent mechanical ventilator, and microgels containing $10 \% \mathrm{w} / \mathrm{v}$ GelMA and different concentrations of nHA nanoparticles $(0 \%-3 \% \mathrm{w} / \mathrm{v})$ laden with osteoinductive pretreated hPDLSCs at $\mathrm{P} 3$ (for 1 week) were transplanted subcutaneously into the dorsal regions of the nude mice. Each group (10\% w/v GelMA with $0 \%, 1 \%, 2 \%$, and $3 \% \mathrm{w} / \mathrm{v} \mathrm{nHA}$ ) consisted of eight-cell material implants. Incisions were closed using 5-0 silk threads (Jinhua Medical Supplies Co., Shanghai, People's Republic of China). Animals were sacrificed 8 weeks later, and the implants were rinsed with DPBS several times followed by fixation for subsequent analysis.

\section{Histological evaluation and SEM of the implants}

To evaluate new bone formation 8 weeks after in vivo transplantation, histological examinations (including hematoxylin and eosin [HE] and Masson's trichrome staining) and SEM analysis of the implants were carried out. Briefly, for histological examinations, implants were harvested, fixed with $4 \%$ paraformaldehyde, and decalcified in $10 \%$ ethylenediaminetetraacetic acid solution at room temperature for $2-3$ weeks. Then, the samples were sliced into $5 \mathrm{~mm}$-thick paraffin-embedded sections and stained with HE or Masson's trichrome stains. ${ }^{25}$ Histological analyses were performed using an Olympus IX81 microscope. For SEM analysis of the implants, similar procedures were performed as described earlier.

\section{Statistical analysis}

All statistical analyses were performed with one-way analysis of variance with Tukey's post hoc test or paired $t$-test accordingly using the GraphPad Prism software (San Diego, CA, USA). Each datum was expressed as mean \pm standard error of mean of at least three independent experiments $(\mathrm{n} \geq 3)$. A $P$-value $<0.05$ was accepted as statistically significant.

\section{Results Characterization of GelMA/nHA microgels}

GelMA/nHA microgel arrays were fabricated through a photolithography strategy. GelMA hydrogel precursor mixed with nHA nanoparticles were polymerized by exposing to UV light (Figure 1A). Here, addition of methacrylate groups to the amine-containing side groups of gelatin can be utilized to make it photocrosslinkable to a hydrogel (Figure 1B). The shape of the hydrogel can be regulated through a designed photomask (diameter 1,000 $\mu \mathrm{m}$ ). Moreover, GelMA is the hydrolysis product of collagen and contains lots of adhesive 
ligands (eg, arginine-glycine-aspartic acid sequences) that promote cell adhesion. The morphology of GelMA/ nHA microgel arrays was well identified by encapsulating fluorescein isothiocyanate-fluorescent beads in microgels (Figure 1C). The microarchitecture of GelMA/nHA microgels was then characterized. SEM images indicated that the pore size of hydrogel decreased with increasing nHA
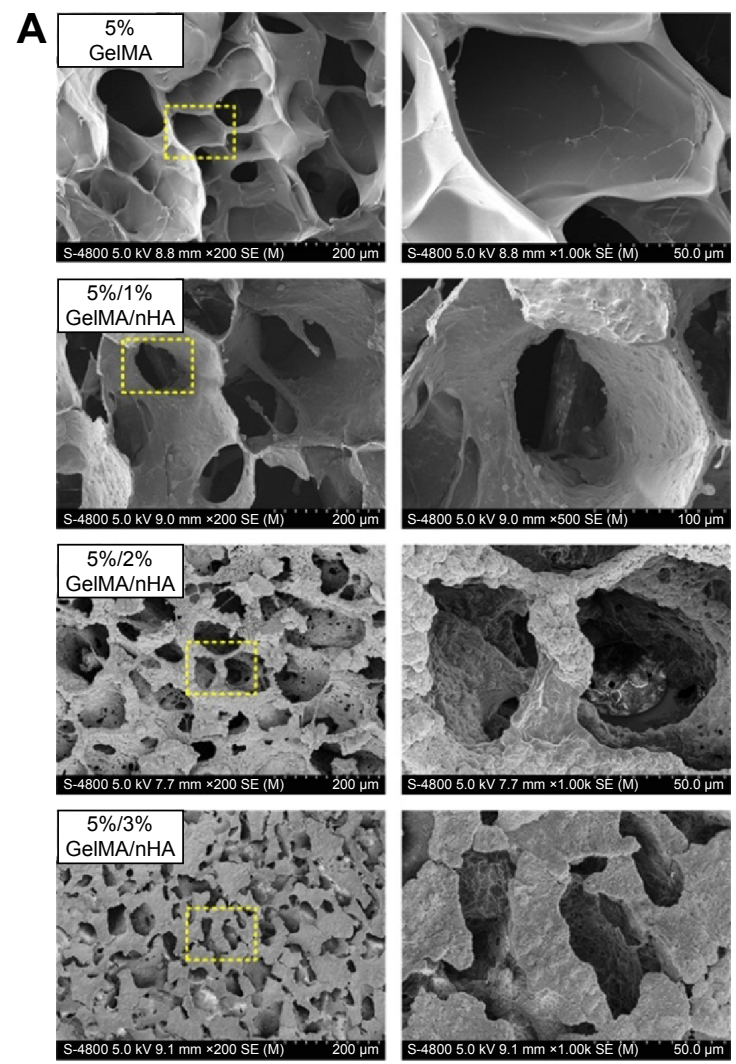

C

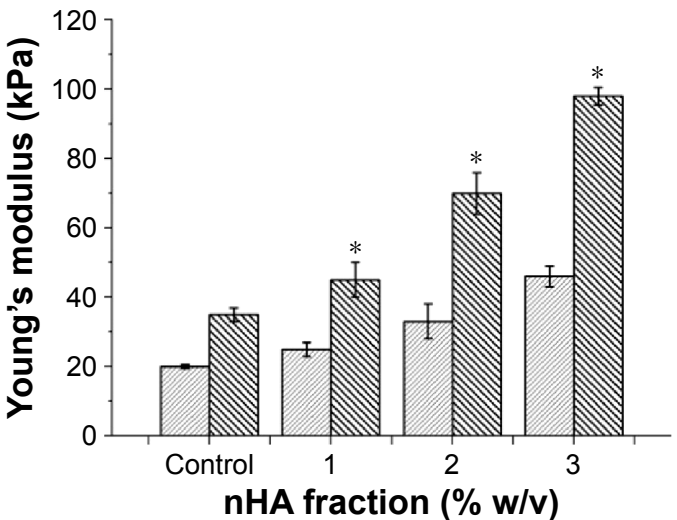

fraction (Figure 2A and B). For the 5\% w/v GelMA group, microgels exhibited a $3 \mathrm{D}$ interconnected pore structure, with pores of size $170 \pm 10 \mu \mathrm{m}$. The wall surface of micropores became rougher with increasing $\mathrm{nHA}$ fraction. The pore size decreased to $15 \pm 3.5 \mu \mathrm{m}$ when GelMA microgels were modified by $3 \% \mathrm{w} / \mathrm{v}$ nHA. SEM images of the $10 \% \mathrm{w} / \mathrm{v}$ GelMA group suggested that the pore size of the microgel

B
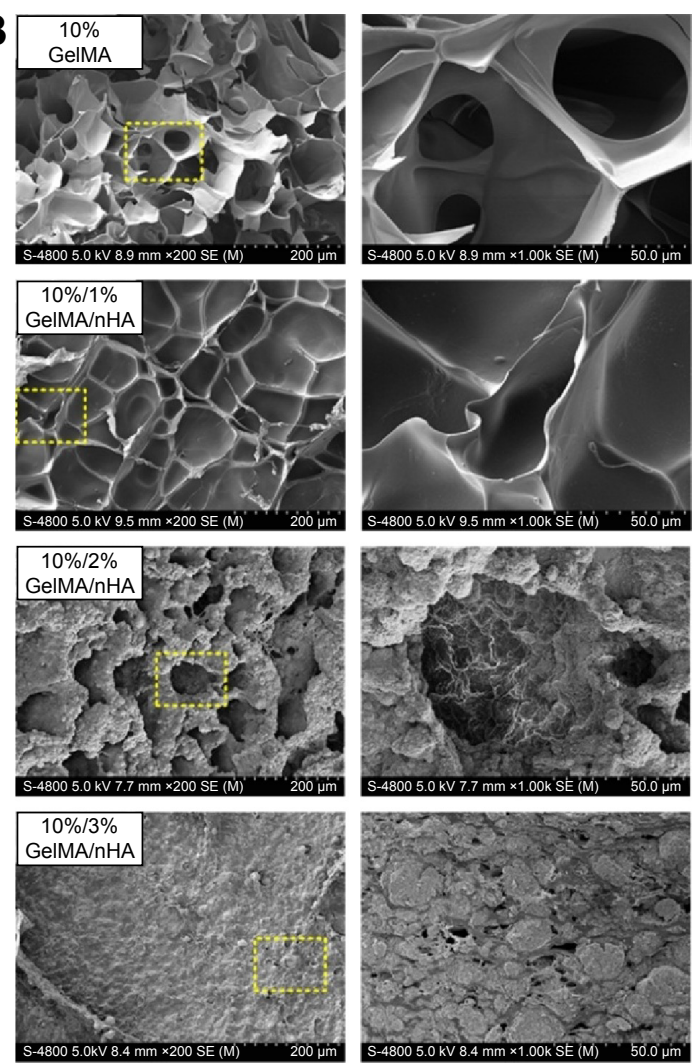

D

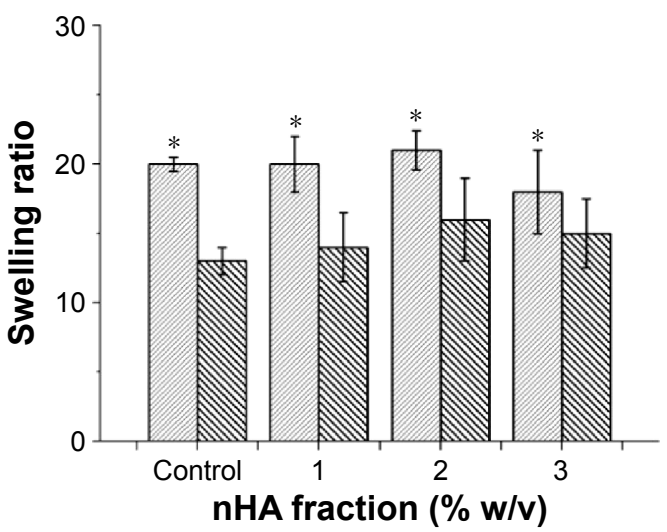

$5 \%$ w/v GelMA $10 \%$ w/v GelMA

Figure 2 Characterization of GelMA/nHA microgels.

Notes: SEM images of $5 \%(\mathbf{A})$ and $10 \%$ (B) GelMA microgels with different $\mathrm{nHA}$ concentrations (from $0 \%$ to $3 \%$ ). Images on the right side are the magnification ( $\times$ I,000) of the yellow dashed squares on the left images $(\times 200)$. (C) Young's modulus of microgels composed of different GelMA concentrations and nHA fractions. Stiffness of microgels was enhanced by increasing both monomer and $\mathrm{nHA}$ concentration $\left({ }^{*} P<0.05\right)$. (D) Swelling properties of GelMA/nHA microgels. The mass swelling ratios are only statistically relevant to GelMA concentrations $(* P<0.05)$. Error bars represent the standard deviation of measurements performed on at least five samples.

Abbreviations: GelMA, gelatin methacrylate; $\mathrm{nHA}$, nanohydroxylapatite; SEM, scanning electron microscopy. 
also decreased with increasing nHA fraction. The pore sizes were $108 \pm 11 \mu \mathrm{m}$ and $20 \pm 2.3 \mu \mathrm{m}$ for the $10 \% \mathrm{w} / \mathrm{v}$ GelMA group (control) and the 10\%/2\% w/v GelMA/nHA group, respectively. nHA nanoparticles significantly aggregated in the $10 \% / 3 \% \mathrm{w} / \mathrm{v}$ GelMA/nHA microgels. We also assessed the biodegradable property of GelMA hydrogel encapsulated with hPDLSCs, which is important for cell growth, penetration, and remodeling of the local microenvironment (Figure S1).

The mechanical properties and swelling ratio of GelMA/ nHA microgels were also tested. The results indicated that the stiffness of microgels was enhanced by increasing both monomer and nHA concentration (Figure 2C). For the control group, Young's modulus was $20 \pm 1.5 \mathrm{kPa}(5 \% \mathrm{w} / \mathrm{v}$ GelMA) and $35 \pm 2.3 \mathrm{kPa}(10 \% \mathrm{w} / \mathrm{v}$ GelMA), respectively. The modulus can be significantly increased when mixed with nHA at a concentration of $3 \% \mathrm{w} / \mathrm{v}(46 \pm 3 \mathrm{kPa}$ for $5 \% \mathrm{w} / \mathrm{v}$ GelMA and $98 \pm 5 \mathrm{kPa}$ for $10 \% \mathrm{w} / \mathrm{v}$ GelMA). Interestingly, the swelling ratio of GelMA/nHA microgels was only related to GelMA monomer fraction (Figure 2D). There was no significant difference between groups with varying nHA concentrations. Here, $10 \%$ w/v GelMA microgel was selected for further studies because of its better mechanical properties, which can meet the mechanical properties of neonatal alveolar bone tissues.

\section{A Live/Dead staining}
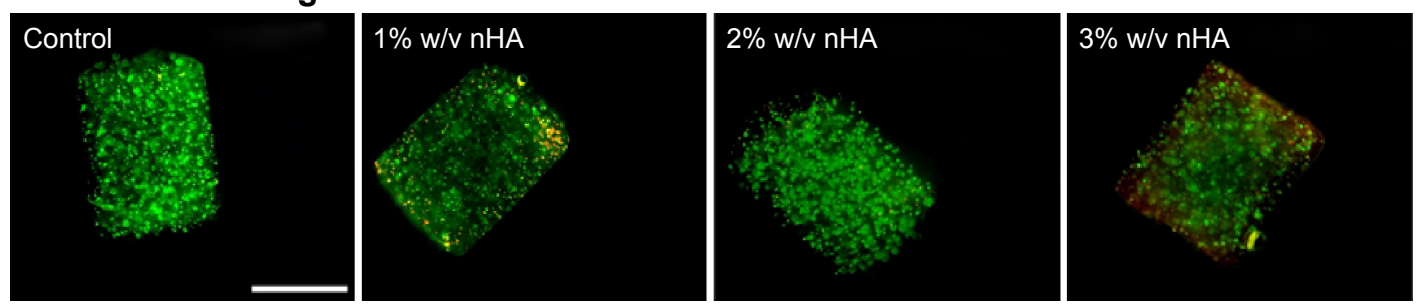

B

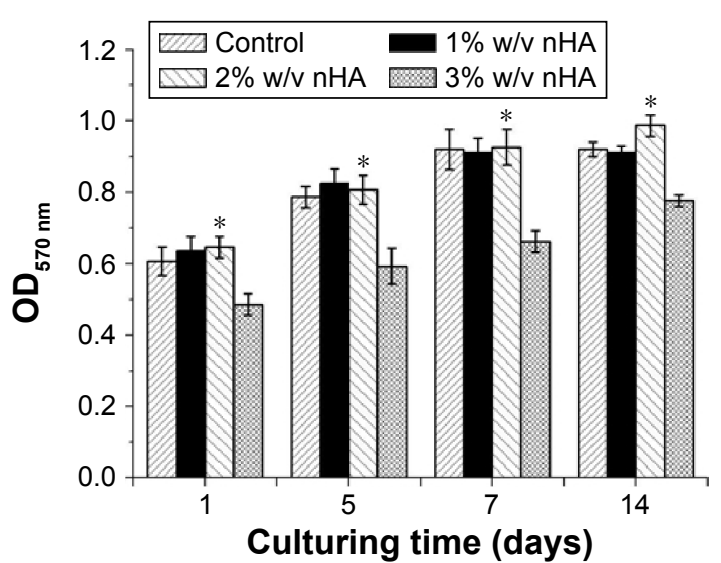

Viability, proliferation, and osteodifferentiation of hPDLSCs in microgels

hPDLSCs were encapsulated in GelMA/nHA microgels and the viability of cells tested using both Live/Dead staining and MTT assay (Figure 3A and B). Fluorescent images indicated that the viability of cells in the $10 \% \mathrm{w} / \mathrm{v}$ GelMA groups after 24 hours encapsulation was not affected when nHA concentration was $1 \% \mathrm{w} / \mathrm{v}$ and $2 \% \mathrm{w} / \mathrm{v}$. For the $3 \% \mathrm{w} / \mathrm{v}$ nHA group, the number of dead cells increased. Similarly, MTT assay results suggested that cell viability significantly decreased in the $3 \% \mathrm{w} / \mathrm{v}$ nHA group. However, cell viability increased with increasing culture time. Cell proliferation in GelMA/nHA microgels was also assessed through BrdU incorporation after 3 days of culture (Figure 3C). The results showed that cells significantly proliferated in the $2 \% \mathrm{nHA}$ group compared to other groups, with a positive BrdU cell percent of $34 \% \pm 4 \%$. The positive cell percent in the $3 \%$ w/v nHA group was only $11 \% \pm 2.5 \%$. We then investigated the osteogenic differentiation potential of hPDLSCs encapsulated in GelMA/nHA microgels using Alizarin Red S staining (Figure 3D). The images suggested that hPDLSCs encapsulated in 2\% nHA exhibited significant mineralization after 10 days of culture compared to microgels with other ratios. Additionally, we evaluated mineralization by

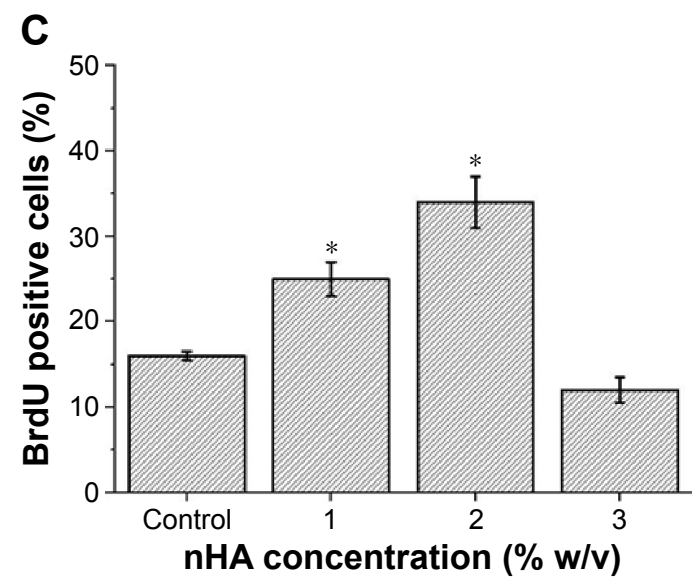

Figure 3 (Continued) 
D
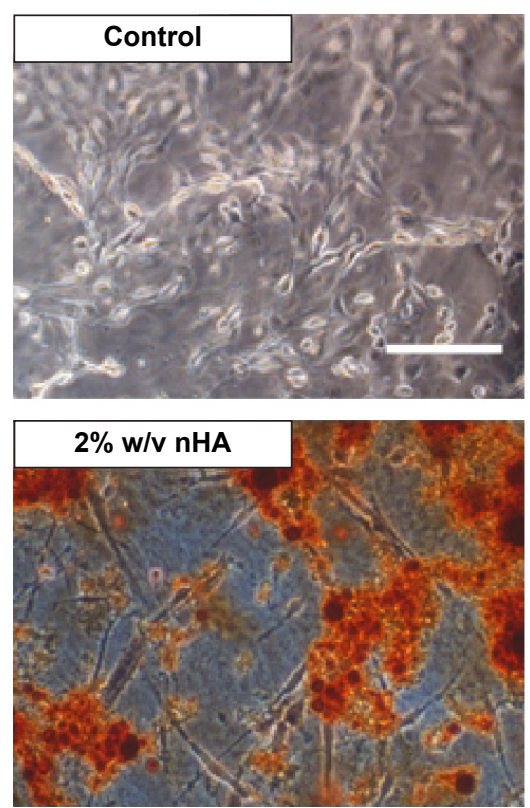

E
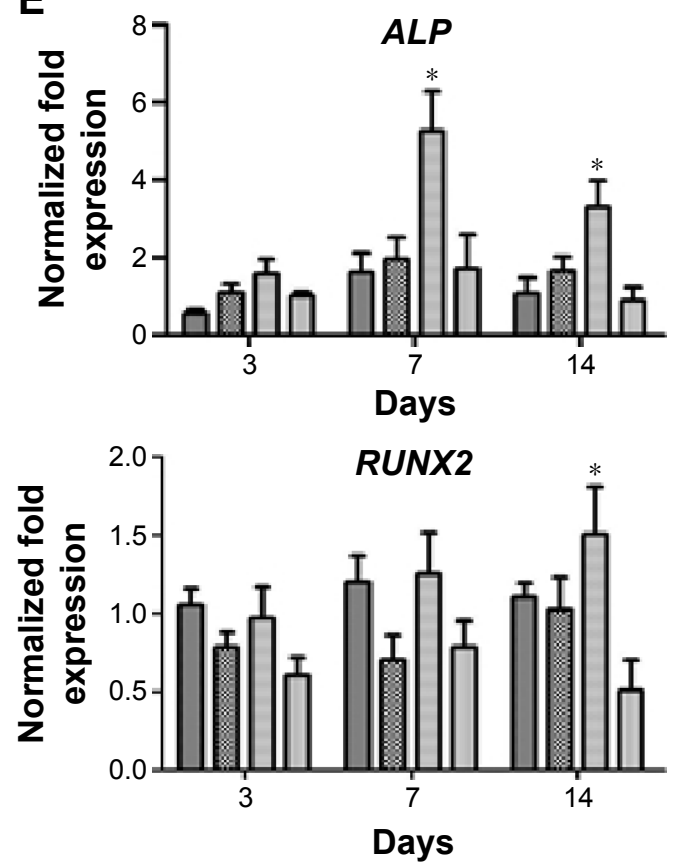
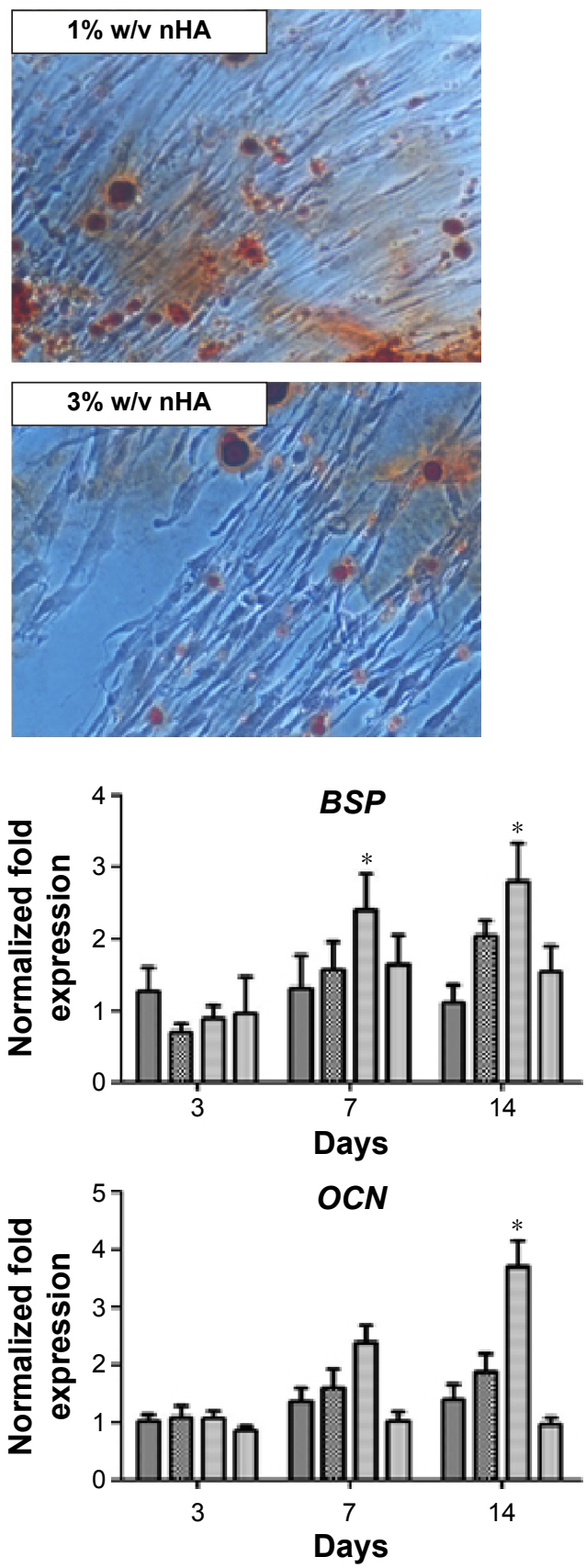

GelMA GelMA/1\% nHA $\square$ GelMA/2\% nHA $\square$ GelMA/3\% nHA

Figure 3 Cell viability, proliferation, and osteodifferentiation potential encapsulated in GelMA/nHA microgels.

Notes: (A) Live/Dead staining showed that cell viability was not jeopardized in the $1 \%$ and $2 \% \mathrm{nHA}$ groups, as compared to the pure GelMA group. However, dead cell number increased in the $3 \% \mathrm{nHA}$ group (scale bar: $800 \mu \mathrm{m}$ ). MTT (B) and BrdU incorporation results (C) showed that hPDLSCs survived and proliferated better in the $2 \%$ $\mathrm{nHA}$ group than in other groups $\left({ }^{* P}<0.05\right)$. (D) Representative images of hPDLSCs encapsulated in microgels of different $\mathrm{nHA}$ concentrations after 10 days of osteoinductive culture (stained with Alizarin Red S staining, scale bar: $100 \mu \mathrm{m}$ ). (E) Relative expressions of $A L P, B S P, R U N X 2$, and $O C N$ of hPDLSCs $(* P<0.05)$. Error bars represent the standard deviation of measurements performed on at least five samples.

Abbreviations: GelMA, gelatin methacrylate; hPDLSCs, human periodontal ligament stem cells; nHA, nanohydroxylapatite; MTT, 3-(4,5-dimethylthiazol-2-yl)-2,5-diphenyl tetrazolium bromide; $A L P$, alkaline phosphatase; BSP, bone sialoprotein; RUNX2, runt-related transcription factor 2; OCN, osteocalcin.

analyzing the expression of osteogenic biomarkers including $A L P, B S P, O C N$, and RUNX2 through RT-PCR (Figure 3E). The results suggested that $2 \% \mathrm{w} / \mathrm{v}$ nHA encapsulation in $10 \%$ w/v GelMA microgels significantly enhanced the osteogenic differentiation of hPDLSCs after 14 days of culture.

\section{In vivo osteogenesis}

To evaluate the in vivo osteogenic potential, the preosteoinduced hPDLSC microgel composite materials were subcutaneously implanted into the backs of nude mice. During the 8 weeks of breeding, all the animals healed uneventfully, and 

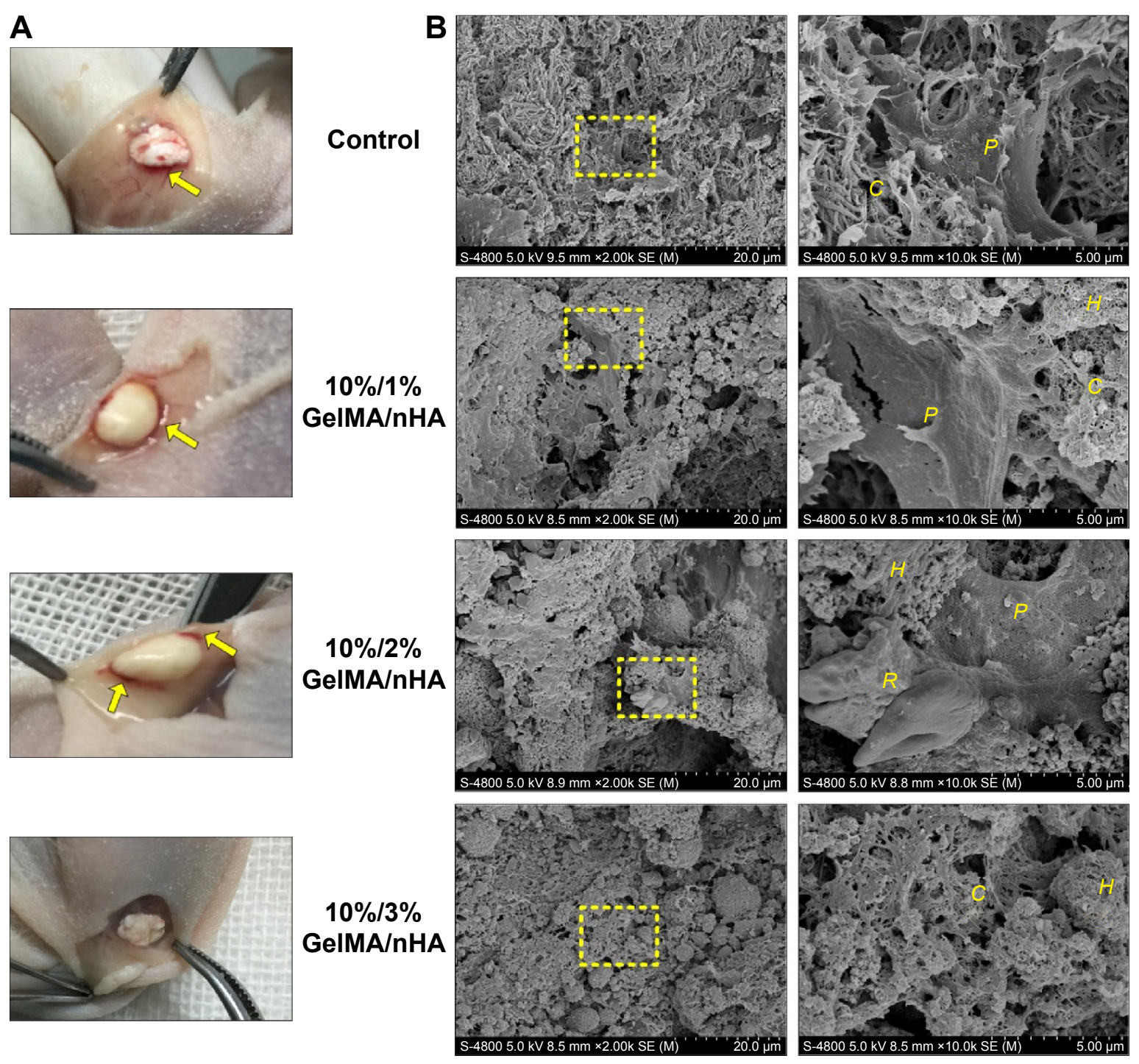

Figure 4 Gross observation and SEM examination of implantation in nude mice after 8 weeks.

Notes: (A) Gross examinations of implants harvested 8 weeks after implantation. Capillaries could be seen in I\%, $2 \% \mathrm{nHA}$, and the control groups (arrows). (B) SEM figures of implants. Images on the right side are the magnification $(\times 1,000)$ of the yellow dashed squares on the left images $(\times 200)$.

Abbreviations: $\mathrm{C}$, collagenous fibers; $\mathrm{H}$, nano-hydroxyapatite; $\mathrm{P}$, periodontal ligament stem cells; $\mathrm{R}$, red blood cells; GelMA, gelatin methacrylate; nHA, nanohydroxylapatite; SEM, scanning electron microscopy.

there was no sign of complications or material exposure at the surgical sites. From the gross view of the implants, we found that all the implants integrated well with the subcutaneous tissue of the nude mice and that vascularization was detected in the control, $1 \%$, and $\% \mathrm{w} / \mathrm{v}$ nHA groups (Figure $4 \mathrm{~A}$ ), and this was further verified by SEM images where red blood cells were found inside the microgels of the $2 \% \mathrm{w} / \mathrm{v}$ nHA (Figure 4B), suggesting that vascularization can be induced within GelMA/nHA microgels. The internal ultrastructure of the implants was demonstrated using SEM. In the control, $1 \%$, and $2 \%$ nHA groups, hPDLSCs penetrated through connective fibers with or without nHA attached and encapsulated, thereby showing good spreading and attachment. However, cells were rarely seen in the $3 \%$ nHA group
(Figure 4B). New periodontal tissue formation was investigated by $\mathrm{HE}$ and Masson's trichrome staining (Figure 5A and B). Both HE and Masson's histological staining results indicated that a certain amount of PDL-like tissue and blood vessels formed in the control, $1 \%$, and $2 \%$ nHA groups. Mineralization can be obtained by increasing nHA fraction in microgels. For the $1 \%$ and $3 \% \mathrm{w} / \mathrm{v}$ nHA group, newly formed tissue constructs showed a lack of cell populations and ECM components such as collagen. This may be caused by the insufficient nHA fraction and stack porosity, as a result of limited space that inhibits cell growth and penetration. Uniform mineralization was seen in the $2 \%$ nHA group, and bone crypts with multinucleated cells (osteoclast-like cells) were initially generated. 
A

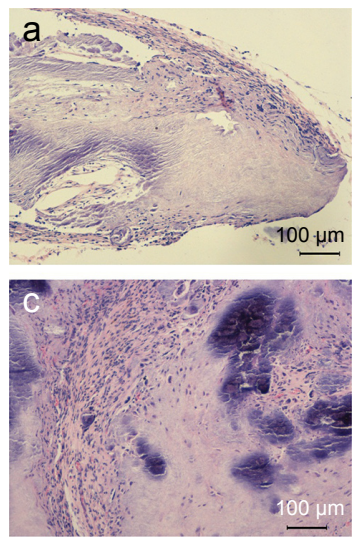

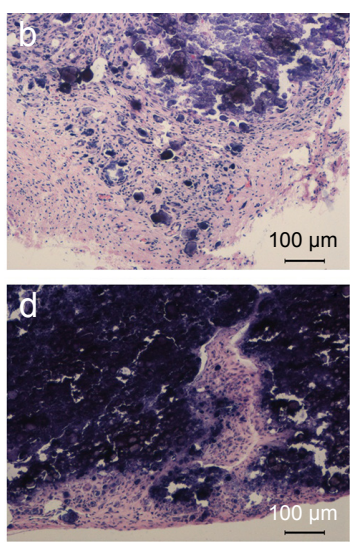

B

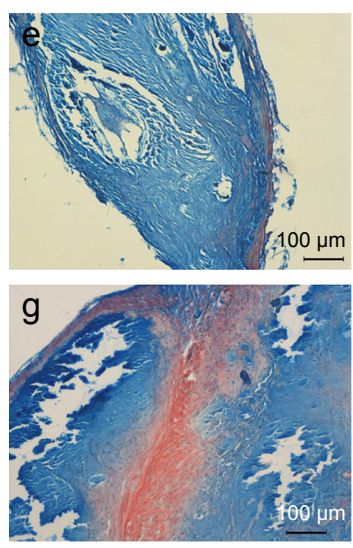

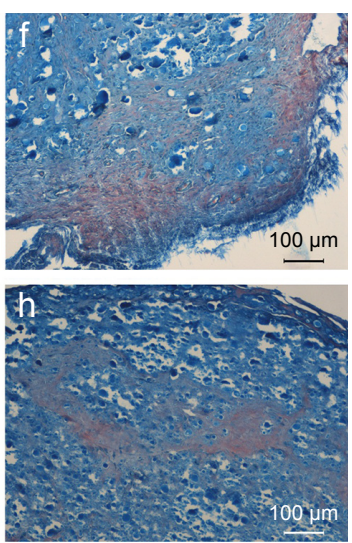

Figure 5 Histological staining images of in vivo ectopic transplantation of hPDLSCs-laden GelMA/nHA microgels.

Notes: (A) HE staining; (B) Masson's trichrome staining. (a) and (e) $10 \%$ GelMA (control), (b) and (f) $10 \% / 1 \%$ GelMA/nHA, (c) and (g) $10 \% / 2 \%$ GelMA/nHA, (d) and (h) 10\%/3\% GelMA/nHA. Scale bar: $100 \mu \mathrm{m}$.

Abbreviations: HE, hematoxylin and eosin; GelMA, gelatin methacrylate; nHA, nanohydroxylapatite; hPDLSCs, human periodontal ligament stem cells.

\section{Discussion}

Great efforts have been made to develop methods for tissue regeneration using 3D scaffolds. ${ }^{26}$ For periodontal tissue regeneration, existing studies have demonstrated that biocompatible scaffolds can promote hPDLSCs adhesion and proliferation..$^{1,2,8}$ However, the fabrication process is low throughput, and the utilized natural scaffolds can hardly meet the sufficient mechanical strength of in vivo periodontal tissues. ${ }^{27,28}$ Additionally, the sample created using the existing method exhibited large sample size with limited diffusion properties, which may affect cell function and make it hard to achieve long-term culture. ${ }^{29,30} \mathrm{GelMA} / \mathrm{nHA}$ microgel arrays provide great potential for periodontal tissue regeneration with sufficient mechanical strength and can also allow for the generation of high-throughput microscale samples rapidly. The structural characterization results suggested that the pore size decreased with increasing GelMA and nHA fraction. nHA incorporation enhanced the topographical properties of the wall surface of interconnected pores, which may promote cell adhesion. ${ }^{31,32}$ Aggregation of nHA nanoparticles occurred when nHA fraction was larger than $2 \% \mathrm{w} / \mathrm{v}$, which may significantly decrease the diffusion of nutrients in microgels and further inhibit cell viability. The mechanical properties of microgels can be enhanced by increasing nHA fraction, which may have resulted from the uniform distribution of nanoparticles in microgels, and the stability of 3D polymer network subsequently increased. The biocompatibility of GelMA/nHA microgels was also investigated. Cell viability, proliferation, and osteogenic differentiation were significantly decreased in the $3 \% \mathrm{w} / \mathrm{v}$ nHA group due to the compact pore structure of the microgels. The nHA fraction was optimized in both in vitro and in vivo experiments. In vivo studies indicated that both mineralization and vascularization occurred in the $10 \% \mathrm{w} / \mathrm{v} / 2 \% \mathrm{w} / \mathrm{v}$ GelMA/nHA microgels. All these results suggested that the $10 \% \mathrm{w} / \mathrm{v} / 2 \% \mathrm{w} / \mathrm{v}$ GelMA/ nHA microgels meet the requirement for tensile strength in bone regeneration and enhanced in vivo new bone formation, compared with the pure $10 \% \mathrm{w} / \mathrm{v}$ GelMA microgels.

\section{Conclusion}

In this study, high-throughput and biocompatible GelMA/ nHA microgel arrays were fabricated using a photolithography strategy. Taken together, the experimental results indicated that the microstructure and mechanical properties can be regulated by changing GelMA and nHA fraction. hPDLSCs encapsulated in GelMA/nHA microgels exhibited high viability, proliferation potential, and strong osteogenic differentiation ability in vitro. Additionally, GelMA/nHA microgels significantly enhanced new bone regeneration in nude mice, suggesting that such microgels hold great potential for periodontal tissue regeneration.

\section{Acknowledgments}

This work was financially supported by the National Natural Science Foundation of China grants (no 81271188 and no 81200823). The authors appreciate Prof Feng Xu and Doctor Yuhui Li from Bioinspired Engineering and Biomechanics Center (BEBC) of Xi' an Jiaotong University for their kind help.

\section{Author contributions}

$\mathrm{YZ}, \mathrm{SB}$, and $\mathrm{XC}$ designed the experiments. XC, BL, and $\mathrm{HL}$ performed the in vitro experiments. XC and SL performed the in vivo experiments. $\mathrm{YZ}$ and $\mathrm{GW}$ interpreted the results 
and revised the paper. $\mathrm{XC}$ wrote the main text of the manuscript. All authors contributed toward data analysis, drafting and revising the paper, and agreed to be accountable for all aspects of the work.

\section{Disclosure}

The authors report no conflicts of interest in this work.

\section{References}

1. Chen X, Wu G, Feng Z, et al. Advanced biomaterials and their potential applications in the treatment of periodontal disease. Crit Rev Biotechnol. 2016;36(4):760-775.

2. Lu H, Xie C, Zhao Y-M, Chen F-M. Translational research and therapeutic applications of stem cell transplantation in periodontal regenerative medicine. Cell Transplant. 2013;22(2):205-229.

3. Steindorff MM, Lehl H, Winkel A, Stiesch M. Innovative approaches to regenerate teeth by tissue engineering. Arch Oral Biol. 2014;59(2): 158-166.

4. Chen F-M, An Y, Zhang R, Zhang M. New insights into and novel applications of release technology for periodontal reconstructive therapies. J Control Release. 2011;149(2):92-110.

5. Rios HF, Lin Z, Oh B, Park CH, Giannobile WV. Cell-and genebased therapeutic strategies for periodontal regenerative medicine. J Periodontol. 2011;82(9):1223-1237.

6. Padial-Molina M, Rios HF. Stem cells, scaffolds and gene therapy for periodontal engineering. Curr Oral Health Rep. 2014;1(1):16-25.

7. Bright R, Hynes K, Gronthos S, Bartold P. Periodontal ligamentderived cells for periodontal regeneration in animal models: a systematic review. J Periodontal Res. 2015;50(2):160-172.

8. Sculean A, Nikolidakis D, Nikou G, Ivanovic A, Chapple IL, Stavropoulos A. Biomaterials for promoting periodontal regeneration in human intrabony defects: a systematic review. Periodontol 2000. 2015;68(1):182-216.

9. Jung IH, Park JC, Kim JC, et al. Novel application of human periodontal ligament stem cells and water-soluble chitin for collagen tissue regeneration: in vitro and in vivo investigations. Tissue Eng Part A. 2012; 18(5-6):643-653.

10. Simmons CA, Alsberg E, Hsiong S, Kim WJ, Mooney DJ. Dual growth factor delivery and controlled scaffold degradation enhance in vivo bone formation by transplanted bone marrow stromal cells. Bone. 2004;35(2): 562-569.

11. Niranjan R, Koushik C, Saravanan S, Moorthi A, Vairamani M, Selvamurugan N. A novel injectable temperature-sensitive zinc doped chitosan/ $\beta$-glycerophosphate hydrogel for bone tissue engineering. Int J Biol Macromol. 2013;54:24-29.

12. Lutolf MP, Weber FE, Schmoekel HG, et al. Repair of bone defects using synthetic mimetics of collagenous extracellular matrices. Nature Biotechnol. 2003;21(5):513-518.

13. Li Y, Poon CT, Li M, Lu TJ, Pingguan-Murphy B, Xu F. Chinesenoodle-inspired muscle myofiber fabrication. Adv Funct Mater. 2015; 25(37):5999-6008.

14. Yue K, Trujillo-de Santiago G, Alvarez MM, Tamayol A, Annabi N, Khademhosseini A. Synthesis, properties, and biomedical applications of gelatin methacryloyl (GelMA) hydrogels. Biomaterials. 2015;73: 254-271.
15. Li Y, Huang G, Gao B, et al. Magnetically actuated cell-laden microscale hydrogels for probing strain-induced cell responses in three dimensions. NPG Asia Mater. 2016;8(1):e238.

16. Li Y, Huang G, Li M, et al. An approach to quantifying 3D responses of cells to extreme strain. Sci Rep. 2016;6:19550.

17. Bertassoni LE, Cardoso JC, Manoharan V, et al. Direct-write bioprinting of cell-laden methacrylated gelatin hydrogels. Biofabrication. 2014; 6(2):024105.

18. Heo DN, Ko W-K, Bae MS, et al. Enhanced bone regeneration with a gold nanoparticle-hydrogel complex. J Mater Chem B. 2014;2(11): 1584-1593.

19. Nichol JW, Koshy ST, Bae H, Hwang CM, Yamanlar S, Khademhosseini A. Cell-laden microengineered gelatin methacrylate hydrogels. Biomaterials. 2010;31(21):5536-5544.

20. Lei M, Li K, Li B, Gao L-N, Chen F-M, Jin Y. Mesenchymal stem cell characteristics of dental pulp and periodontal ligament stem cells after in vivo transplantation. Biomaterials. 2014;35(24):6332-6343.

21. Zhang J, An Y, Gao LN, Zhang YJ, Jin Y, Chen FM. The effect of aging on the pluripotential capacity and regenerative potential of human periodontal ligament stem cells. Biomaterials. 2012;33(29):6974-6986.

22. Moshaverinia A, Chen $\mathrm{C}, \mathrm{Xu} \mathrm{X}$, et al. Bone regeneration potential of stem cells derived from periodontal ligament or gingival tissue sources encapsulated in RGD-modified alginate scaffold. Tissue Eng Part A. 2013; 20(3-4):611-621.

23. Kwon JS, Kim SW, Kwon DY, et al. In vivo osteogenic differentiation of human turbinate mesenchymal stem cells in an injectable in situforming hydrogel. Biomaterials. 2014;35(20):5337-5346.

24. Clark JD, Gebhart GF, Gonder JC, Keeling ME, Kohn DF. The 1996 guide for the care and use of laboratory animals. ILAR J. 1997;38(1): 41-48.

25. Yang H, Gao L-N, An Y, et al. Comparison of mesenchymal stem cells derived from gingival tissue and periodontal ligament in different incubation conditions. Biomaterials. 2013;34(29):7033-7047.

26. Lu T, Li Y, Chen T. Techniques for fabrication and construction of three-dimensional scaffolds for tissue engineering. Int J Nanomedicine. 2013;8:337.

27. Wagner DE, Bonenfant NR, Sokocevic D, et al. Three-dimensional scaffolds of acellular human and porcine lungs for high throughput studies of lung disease and regeneration. Biomaterials. 2014;35(9):2664-2679.

28. Sullivan DC, Mirmalek-Sani S-H, Deegan DB, et al. Decellularization methods of porcine kidneys for whole organ engineering using a highthroughput system. Biomaterials. 2012;33(31):7756-7764.

29. Loh QL, Choong C. Three-dimensional scaffolds for tissue engineering applications: role of porosity and pore size. Tissue Eng Part B Rev. 2013; 19(6):485-502.

30. Matsusaki M, Case CP, Akashi M. Three-dimensional cell culture technique and pathophysiology. Adv Drug Deliv Rev. 2014;74:95-103.

31. Liu H, Xu GW, Wang YF, et al. Composite scaffolds of nanohydroxyapatite and silk fibroin enhance mesenchymal stem cell-based bone regeneration via the interleukin 1 alpha autocrine/paracrine signaling loop. Biomaterials. 2015;49:103-112.

32. Meng Z, Chitrakar C, Gaharwar AK, Yakovlev VV. Reinforcement of osteogenesis with nanofabricated hydroxyapatite and GelMA nanocomposite. Paper presented at SPIE BiOS 2015, Photonic Therapeutics and Diagnostics XI, 930340; February 26; 2015; San Francisco, CA. 


\section{Supplementary material}

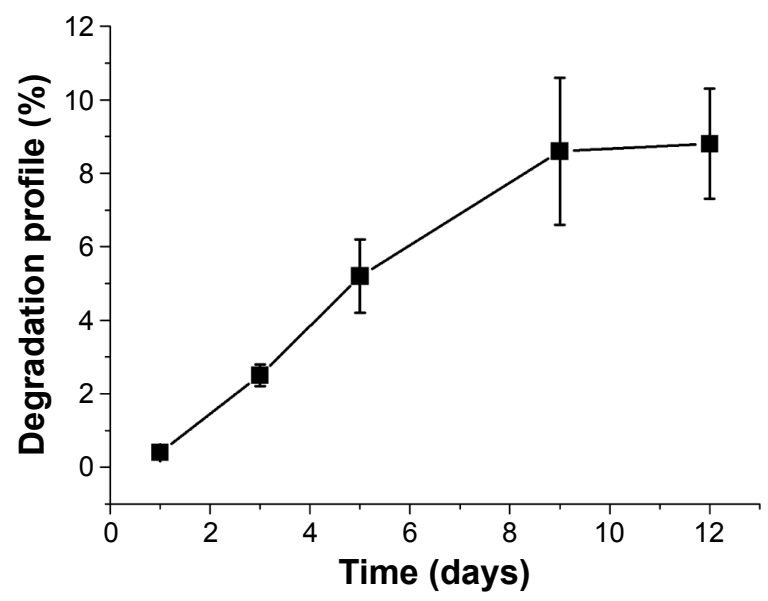

Figure SI Evaluation of degradation profile of cell-laden GelMA hydrogels.

Abbreviation: GelMA, gelatin methacrylate.

\section{Publish your work in this journal}

The International Journal of Nanomedicine is an international, peerreviewed journal focusing on the application of nanotechnology in diagnostics, therapeutics, and drug delivery systems throughout the biomedical field. This journal is indexed on PubMed Central, MedLine, CAS, SciSearch ${ }^{\circledR}$, Current Contents ${ }^{\circledR} /$ Clinical Medicine,
Journal Citation Reports/Science Edition, EMBase, Scopus and the Elsevier Bibliographic databases. The manuscript management system is completely online and includes a very quick and fair peer-review system, which is all easy to use. Visit http://www.dovepress.com/ testimonials.php to read real quotes from published authors. 